\title{
Chromosome 1 Abnormalities Predict Shortened Progression Free and Overall Survival in Patients with High Risk Multiple Myeloma Undergoing Autologous Hematopoietic Cell Transplantation, a Retrospective Analysis
}

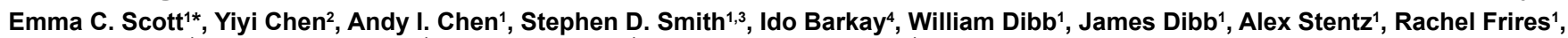
Matthew Siegel' ${ }^{1}$, Phoebe Trubowitz ${ }^{1}$, Eva Medvedova ${ }^{1}$ and Richard T. Maziarz ${ }^{1}$

${ }^{1}$ Center for Hematologic Malignancies, The Knight Cancer Institute, Oregon Health Sciences University, USA

${ }^{2}$ The Department of Public Health and Preventative Medicine, Oregon Health and Sciences University, USA

${ }^{3}$ Fred Hutchinson Cancer Research Center, The University of Washington, USA

${ }^{4}$ The Department of Internal Medicine, Oregon Health Sciences University, USA

\begin{abstract}
Abnormalities of chromosome (ch)1 have been shown to be significant adverse prognostic factors in multiple myeloma (MM) but they have not yet been systematically studied in patients undergoing autologous hematopoietic cell transplantation (auto-HCT). The aim of this study was to determine whether patients with high-risk MM and ch1 abnormalities (1q gain, $1 \mathrm{p}$ deletion, translocations of ch1) constitute a highest risk group compared to a contemporaneous cohort of high-risk MM patients without ch1 abnormalities. 232 patients (169 induction, 63 salvage) with MM and at least one recognized high-risk feature met criteria for inclusion. The presence of a ch1 abnormality $(n=15)$ was highly significant in patients undergoing salvage autologous $\mathrm{HCT}(n=6)$ for predicting shorter PFS ( $p<0.001 ; \mathrm{HR}=22.93 ; 95 \% \mathrm{Cl}: 4.94-106.48$ ), and OS ( $p=0.0002 ; \mathrm{HR}=21.22 ; 95 \% \mathrm{Cl}: 1.18-14.98$ ). Median PFS and OS for those with a ch1 abnormality and del $13 q(n=7)$ were 4.76 and 9.43 months, with ch1 abnormality and no del $13 \mathrm{q}(\mathrm{n}=8)$ were 16.79 and 35.22 months respectively, and for those Without cytogenetic abnormalities, 24.44 and 57.03 months respectively. Based upon the impact of ch1 abnormalities on auto-HCT outcomes in this study, further investigation in larger series is warranted.
\end{abstract}

Keywords: Multiple myeloma; Chromosome 1; Stem cell transplant

\section{Introduction}

Abnormalities of chromosome 1 (ch1) are common in hematological malignancies and are among the most common structural aberrations found in MM [1,2]. Abnormalities of both the long arm (1q gain) and short arm (1p loss) have been shown to be significant adverse prognostic factors in myeloma [3-8] but they have not yet been included in the new IMWG molecular cytogenetic classification [9], or other large myeloma centers' prognostic criteria such as the Mayo Clinic mSMART criteria [10].

However, according to the 2014 NCCN guidelines, the FISH panel for prognostic estimation should also include ch1 amplification [11].

The literature contains several small cohorts limited to one or few institutions and some studies have failed to confirm the overriding negative prognostic association with chl amplification detected by FISH, thus controversy regarding the impact of ch1 abnormalities exists [6,12]. In particular, the impact of ch1 abnormalities on autoHCT outcomes has been associated with adverse survival $[13,14]$, but has not been widely or systematically studied.

Regarding specific ch1 aberrations encountered, the short arm of ch1 is most often associated with deletions and the long arm of ch1 with amplifications. The majority of these abnormalities involve rearrangements located in the peri-centromeric regions and frequently in the form of jumping translocations.

One of the genes mapped at 1q21 is CKS1B, a member of the highly conserved cyclin kinase subunit 1 (CKS1) protein family that interacts with cyclin dependent kinases (Cdks) and plays a critical role in cell cycle progression [15].

CKS1 is an essential cofactor for efficient Skp2-dependent ubiquitination of p27Kip1 [16]. CKS1B overexpression is correlated with low p27 expression and adverse survival in several human malignancies.

Its presence in $\mathrm{MM}$ is associated with a higher risk of progression and the incidence is higher in relapsed than in newly diagnosed patients [17]. Although less commonly described, deletions of CDKN2C at 1p32 impact G1/S transition resulting in poor outcomes [13].

The aim of this study was to describe MM patients with any high risk characteristic that underwent auto-HCT and to compare outcomes between various high risk groups. Specifically, we aimed to identify whether ch1 abnormalities (including 1q gain, $1 \mathrm{p}$ deletion and translocations of chromosome 1 with other partners) constituted a 'highest' risk group that may benefit from investigation of alternative treatment strategies in future studies.

\section{Patients and Methods}

A retrospective cohort study of MM patients with any high risk feature, who received an auto-HCT as induction or salvage treatment at OHSU between $01 / 01 / 2001$ and 12/31/2011 was performed after

${ }^{*}$ Corresponding author: Emma C. Scott, MD, The Center for Hematologic Malignancies, Oregon Health and Science University, 3181 SW Sam Jackson Park Road, Portland, Oregon, USA 97239, Tel: 503 494-8311; E-mail: scottem@ohsu.edu

Received November 22, 2014; Accepted January 07, 2015; Published January 15,2015

Citation: Scott EC, Chen Y, Chen Al, Smith SD, Barkay I, et al. (2015) Chromosome 1 Abnormalities Predict Shortened Progression Free and Overall Survival in Patients with High Risk Multiple Myeloma Undergoing Autologous Hematopoietic Cell Transplantation, a Retrospective Analysis. J Blood Lymph 5: 131. doi:10.4172/2165-7831.1000131

Copyright: $\odot 2015$ Scott EC, et al. This is an open-access article distributed under the terms of the Creative Commons Attribution License, which permits unrestricted use, distribution, and reproduction in any medium, provided the original author and source are credited. 
Citation: Scott EC, Chen Y, Chen AI, Smith SD, Barkay I, et al. (2015) Chromosome 1 Abnormalities Predict Shortened Progression Free and Overall Survival in Patients with High Risk Multiple Myeloma Undergoing Autologous Hematopoietic Cell Transplantation, a Retrospective Analysis. J Blood Lymph 5: 131. doi:10.4172/2165-7831.1000131

Page 2 of 7

obtaining Institutional Review Board approval in keeping with the declaration of Helsinki.

Subjects were identified using the CIBMTR database, institutional databases as well as from individual patient medical records. High risk features are defined as cytogenetically detected deletion (del) 13 , cytogenetically detected chromosome 1 abnormality, cytogenetic or FISH detection of del 17p, $\mathrm{t}(4 ; 14)(\mathrm{p} 16 ; \mathrm{q} 32), \mathrm{t}(14 ; 16)(\mathrm{q} 32 ; \mathrm{q} 23)$, $\mathrm{t}(14 ; 20)(\mathrm{q} 32 ; \mathrm{q} 11)$; plasmablastic or anaplastic morpholology, multiple extramedullary plasmacytomas, achieving less than a partial remission with 2 consecutive lines of therapy, International Staging 112 System (ISS) stages II and III, Salmon-Durie staging system stages 2 and 3.

These particular features were included due to the wide acceptance of their significance in the literature. Subjects with standard or good risk cytogenetics or FISH profiles; ISS stage 1 and S-D category 1 were excluded if they did not have a co-existing high risk feature.

To delineate the impact of different genomic groups on PFS and OS, the following groups were formed based on cytogenetic and or FISH findings: group 1 (no abnormality), group 2 comprised all other well-known poor risk cytogenetic factors (del 13q by cytogenetics, $t$ $(4 ; 14), t(14 ; 16), t$ (14;20), del 17p), group 3 comprised of chromosome 1 abnormalities (that occur alone or together with other genomic abnormalities except del 13q) and group 4 comprised of chromosome 1 abnormalities occurring together with del $13 \mathrm{q}$ by cytogenetics).

These particular groups were formed to compare well known high and standard risk genomic profiles to those with ch1 abnormalities. The co-occurrence of ch1 abnormalities with del 13q deletion was most common and we thus decided to explore the significance of this relationship by creating group 4 .

\section{Statistical Analysis}

Descriptive statistical analysis was conducted to summarize overall response rate, relapse rate, progression free survival (PFS), overall survival (OS), patients' individual and clinical characteristics and cytogenetic and FISH profiles, pre-HSCT treatment regimens and post- HSCT maintenance therapy.

PFS, OS and the time to relapse were all calculated from the date of stem cell infusion until the date of progression or death. Patients alive without progression were censored on the date of last follow up for PFS. OS was until the date of death due to any cause and living patients were censored on the last follow up date the patient was known to be alive.

Kaplan-Meier method was used to estimate the distribution of PFS and OS. A log-rank test was used to assess whether the survival function differed across the groups [18]. Univariable and multivariable logistic regression models were fitted to assess the association between overall response rate/relapse rate and risk factors measured prior to the time of transplant.

Univariable and multivariable cox regression models were fitted to evaluate the association between each of the survival endpoints (PFS and OS) and the set of covariates that are the same for the logistic regression models. Final multivariable models were determined based on Akaike's information criterion (AIC) in combination with clinical judgment. Results were considered significant if the P-value was less than or equal to 0.05 . The statistical analysis was performed using SAS Version 9.4 (SAS Institute, Cary, NC, USA).

\section{Results}

A total of 232 subjects were identified and analyzed for this study. Patient characteristics, high risk features, pre-auto-HCT treatment regimens, indication for auto-HCT, pre-auto-HCT treatment characteristics and post-auto-HCT maintenance therapies are listed in (Table 1).

A total of 207 subjects had cytogenetic and/or FISH results. Among the 207 subjects, 55 received a salvage transplant and 152 an induction transplant. Among these, chromosome 1 abnormalities were detected by routine cytogenetic testing in six subjects that underwent salvage transplant and nine subjects that underwent induction transplant. There were 3 balanced translocations, 3 losses of 1q, 6 losses of 1p, 2 gains of $1 \mathrm{q}$ and 1 balanced translocation with gain of $1 \mathrm{q}$ (Table 2).

\section{Treatment Characteristics}

\section{Stem cell transplant}

One auto-HCT per patient was included for analysis. Five subjects underwent a tandem induction auto-HCT. In any single patient, if two transplants were performed $(n=12)$, the second (salvage) transplant was included for analysis. The preparative regimen was Melphalan 200 $\mathrm{mg} / \mathrm{m}^{2}$.

\section{Pre-transplant induction therapy}

Details of the pre-transplant chemotherapy regimens were available in 203 patients. The median number of pre-transplant therapy was 2 (range 1-9).

\section{Post-transplant maintenance therapy}

Details of post auto-HCT maintenance therapy were available in 152 patients. The majority (95 patients) did not receive maintenance therapy, while 27 received lenalidomide, 3 bortezomib, 11 steroids and 16 received thalidomide.

\section{Response and relapse rates post auto-HCT}

Using the International Working Group 183 Criteria, overall response rate (ORR) for the whole group was $81.0 \%$ (95\% CI: 75.4 85.9).

Complete remission (CR) occurred in $33.2 \%$, very good partial remission (VGPR) in $31.9 \%$, partial remission (PR) in $16.0 \%$, minor response $(\mathrm{MR})$ in $1.3 \%$, stable disease $(\mathrm{SD})$ in $5.2 \%$, progressive disease (PD) in $5.2 \%$.

The best response was unknown in $6.0 \%$ of cases. (Table 3) demonstrates results for induction and salvage cohorts.

\section{Progression free and overall survival}

With a median follow up of 44.5 months, 80 (34.5\%) patients in this cohort had died. Among all patients who had valid relapsing information 105 (54.4\%, 193 95\% CI: 38.4-52.9) had relapsed disease.

For the entire group, median PFS and OS was 22.0 (95\% CI: 19.5, 29.4) and 51.8 (95\% CI: 35.2, 63.4) months respectively.

The 2 and 5-year PFS rates were 61.2\% (95\% CI: 54.9, 67.5) and $51.7 \%$ (95\% CI: 45.3, 58.2) respectively.

The 2 and 5 year OS rates were $81.5 \%$ (95\% CI: 76.5, 86.5) and $69.4 \%$ (95\% CI: 63.5, 75.3). (Table 2) demonstrates corresponding results for induction and salvage cohorts. 
Citation: Scott EC, Chen Y, Chen Al, Smith SD, Barkay I, et al. (2015) Chromosome 1 Abnormalities Predict Shortened Progression Free and Overall Survival in Patients with High Risk Multiple Myeloma Undergoing Autologous Hematopoietic Cell Transplantation, a Retrospective Analysis. J Blood Lymph 5: 131. doi:10.4172/2165-7831.1000131

\begin{tabular}{|c|c|c|}
\hline \multirow{2}{*}{\begin{tabular}{|l|} 
Total number of subjects $\mathbf{n}$ \\
Age at transplantation (years) \\
median (range)
\end{tabular}} & \multicolumn{2}{|l|}{232} \\
\hline & & $60(30-79)$ \\
\hline \multirow[t]{2}{*}{ Gender: n (\%) } & Male & $151(65.1)$ \\
\hline & Female & $81(34.9)$ \\
\hline \multirow[t]{3}{*}{ ISS Stage: $(n=168)$} & I & $\begin{array}{c}168 \\
50(29.8)\end{array}$ \\
\hline & II & $75(44.64)$ \\
\hline & III & $43(25.6)$ \\
\hline \multirow[t]{3}{*}{ Salmon-Durie Stage: $(n=167)$} & I & $\begin{array}{c}167 \\
3(1.8)\end{array}$ \\
\hline & II & $46(27.5)$ \\
\hline & III & $118(70.7)$ \\
\hline \multirow{7}{*}{ Genetic abnormality: $(n=207)$} & del $(13 q)$ cytogenetics & $\begin{array}{c}207 \\
20(9.7)\end{array}$ \\
\hline & $t(4 ; 14)$ & $19(9.2)$ \\
\hline & $\operatorname{del}(17 p)$ & $13(6.3)$ \\
\hline & $t(14 ; 16)$ & $3(1.4)$ \\
\hline & $t(14 ; 20)$ & $1(0.5)$ \\
\hline & chromosome 1 abnormality & $15(7.3)$ \\
\hline & no abnormality & $154(74.4)$ \\
\hline Plasmablastic morphology: $\mathrm{n}(\%)$ & & $6(2.6)$ \\
\hline $\begin{array}{l}\text { Multiple extra medullary } \\
\text { plasmacytomas: } n(\%)\end{array}$ & & $14(6.0)$ \\
\hline Prior therapies median (range) & & $2(0-9)$ \\
\hline \multirow{9}{*}{$\begin{array}{l}\text { Pre- transplant regimens } \\
(n=204)\end{array}$} & Bortezomib containing & $86(42.1)$ \\
\hline & Lenalidomide containing & $69(33.8)$ \\
\hline & VRD & $16(7.8)$ \\
\hline & VAD & $64(31.4)$ \\
\hline & Thalidomide containing & $63(30.9)$ \\
\hline & Doxil containing & $15(7.4)$ \\
\hline & HyperCVAD & $9(4.4)$ \\
\hline & DCEP & $5(2.6)$ \\
\hline & Cyclophosphamide containing & $12(5.9)$ \\
\hline $\begin{array}{l}<P R \text { to } 2 \text { conventional lines of } \\
\text { therapy prior to HSCT: } n(\%)\end{array}$ & & $18(7.8)$ \\
\hline \multirow[t]{3}{*}{ Indication for auto- HCT: n (\%) } & \multirow{2}{*}{ Induction } & 232 \\
\hline & & $169(72.8)$ \\
\hline & Salvage & $63(27.2)$ \\
\hline \multirow{6}{*}{ Maintenance: $\mathrm{n}(\%)$} & \multirow{2}{*}{ None } & 152 \\
\hline & & $95(62.1)$ \\
\hline & Lenalidomide & $26(17.1)$ \\
\hline & Bortezomib & $3(2.0)$ \\
\hline & Steroids & $10(6.6)$ \\
\hline & Thalidomide & $16(10.5)$ \\
\hline
\end{tabular}

Table 1: Patient and clinical characteristics $\mathrm{N}=232$. Abbreviations: ISS (International Staging System); t (translocation); Bortezomib, lenalidomide and dexamethasone (VRD); vincristine, adriamycin, dexamethasone (VAD); fractionated cyclophosphamide, vincristine, Adriamycin and dexamethasone (Hyper CVAD), dexamethasone, cyclophosphamide, etoposide, and cisplatin (DCEP), Del (deletion)

\section{Univariable and multivariable analyses of high risk factors associated with shorter PFS and OS}

Cox regression models were fitted for each variable listed in (Table 1). The presence of a ch1 abnormality and achieving less than a PR with 2 consecutive lines of therapy prior to transplant were statistically the most significant factors independently associated with shorter PFS and OS in this cohort of high risk subjects.

The ISS and Salmon-Durie staging 206 systems, increased number of prior therapies, del $17 \mathrm{p}, \mathrm{t}(4 ; 14), \mathrm{t}(14 ; 16), \mathrm{t}(14 ; 20)$, del
$13 q$ by cytogenetics, multiple $(>2)$ extramedullary plasmacytomas, plasmablastic morphology were not significantly independently associated with shorter PFS or OS in this cohort of high risk patients (Table 4).

The final multivariable models for PFS for salvage ASCT found chromosome 1 (HR 22.93; p<0.001; 95\% CI:4.94-106.47) and Salmon

\begin{tabular}{|c|c|c|c|c|}
\hline & Chromosome & & Complete cytogenetic report & \\
\hline 1 & $\begin{array}{l}\text { Balanced } \\
\text { translocation }\end{array}$ & $t(1 ; 10)$ & $\begin{array}{l}47, X,-X, t(1 ; 10)(q 21 ; q 26),+5, \operatorname{der}(5) \\
\text { t(5;8)(p13;q13), } \\
+7, \operatorname{der}(8) t(5 ; 8)(p 13 ; q 13) \operatorname{add}(5)(p 15), \\
\text { add(12)(p1?2),add(13)(q2?2),del(14) } \\
(\text { q24),t(14;17)(q11.2; } 11.2), \operatorname{add}(15) \\
(q ? 21),+19,-20[2] / 46, X X[25]\end{array}$ & Salvage \\
\hline 2 & & $t(1 ; 10)$ & $\begin{array}{l}49 \sim 51, X,-X, t(1 ; 10) \\
(p 13 ; q 24),+5, \text { add }(8)(q 22),+9,- \\
13, \text { add }(14)(q 32),+15,+15,+16, \\
+19,+21[c p 4] / 46, X X[15]\end{array}$ & Induction \\
\hline 3 & & $t(1 ; 14)$ & Not available & Induction \\
\hline 4 & Loss of $1 q$ & $\begin{array}{l}1 \mathrm{q} 12 \\
\mathrm{t}(1 ; 19)\end{array}$ & $\begin{array}{l}51, X,-X, \text { del }(1)(q 12),+ \text { del }(1),- \\
2,+3,+5,+7,+\operatorname{add}(9)(p ? 22),-13,+15, \\
+\operatorname{der}(19) t(1 ; 19)(q 21 ; p 13.3) \\
\text { x2[1]/46,XX[24] }\end{array}$ & Salvage \\
\hline 5 & & $1 \mathrm{q} 32$ & $\begin{array}{l}52, X X, \operatorname{del}(1)(q 32),+3, \operatorname{der}(3) t(3 ; ?) \\
\text { (q12;?), } \\
\text { del(5)(q31),+6,-7,+9,del(12) } \\
\text { (p12),-13,+15,+15,der(16)add(16) } \\
\text { (p11.2)del(16)(q24),add(17) } \\
\text { (p11.2),+21,+mar[cp4]/46,XX[21] }\end{array}$ & Induction \\
\hline 6 & & $\begin{array}{l}1 \mathrm{q} 43 \\
1 \mathrm{q} 32\end{array}$ & $\begin{array}{l}44, X, \operatorname{der}(X ; 1) t(p 11.2 ; p 36.3) \operatorname{del}(X) \\
\text { (p11.2) } \\
\text { add(1)(q43),del(1)(q32),del(2) } \\
\text { (p23),-3, del(6)(q11.2q11.2),del(10) } \\
(\mathrm{p} 11.2 \mathrm{p} 14), \\
\text { add(11)(q23),del(13)(q14q22) } \\
\text { x2,add(14)(q32),- } \\
15, \text { add(16)(p11.2),add(17)(p11.2),- } \\
18, \operatorname{add}(20)(\mathrm{p} 12),-21,-22,+3 \operatorname{mar}[5]\end{array}$ & Induction \\
\hline 7 & Loss of $1 p$ & 1p13-p32 & $\begin{array}{l}52, \mathrm{XY}, \operatorname{del}(1)(? \mathrm{p} 32 ? 13), \operatorname{dic}(1 ; 11) \\
(\mathrm{p} ? 13 ; \mathrm{q} ? 25),+5, \\
\text { add(6)(q?15),+del(6)(p22),+6,+7,-- } \\
8,+9,-11, \text { add(12)(p1?1.2),del(13) } \\
\text { (q?14),+15, } \\
\text { add(16)(q2?4),+18,+19,+21,-- } \\
\text { 22[cp5]/46,XY[14] }\end{array}$ & Salvage \\
\hline 8 & & $1 \mathrm{p} 13$ & $\begin{array}{l}\text { 41 42,X, -Y, add(1)(p13),+der(3)t(3;8) } \\
\text { (p1?1;q1?2), } \\
\text { add(4)(q2?5),add(5) } \\
\text { (p1?2),add(6)(q1?2),-8,+11,-- } \\
13, \text { add(13)(p11.1),-14,der(16) } \\
\text { t(16;17)(q12-13;q1?1.2),-17,- } \\
\text { 18,+mar1[cp3]/46 49,idem,--add(1) } \\
\text { (p13),+6,-add(6)(q1?2),+7,+add(8) } \\
\text { (q?21),+11,-add(13)(p11.2),+del(13) } \\
\text { (q1?2q22),+14,+15[cp7]/ } \\
46, X Y[10]\end{array}$ & Salvage \\
\hline 9 & & $\begin{array}{l}\text { 1p36.1 } \\
1 \mathrm{p} 22\end{array}$ & $\begin{array}{l}\text { 40,X,-X,add(1)(p3?6.1),add(1) } \\
\text { (p?22),add(2)(p?13),-5, add(6)(p22),- } \\
8, \operatorname{add}(9)(q ? 32),-13,-13, \operatorname{add}(16) \\
\text { (p?11.2),add(21)(q22),-22,add(22) } \\
\text { (q13)[cp6]/46,XX[24] }\end{array}$ & Induction \\
\hline 10 & & $\begin{array}{l}1 \mathrm{p} 22 \\
1 \mathrm{p} 22\end{array}$ & $\begin{array}{l}49, X,-Y, \text { add }(1)(p ? 22),+\operatorname{add}(1) \\
(p ? 22), \operatorname{del}(8)(q ? 13 q 22),+11,-13,+15,- \\
18,+21,+1 \sim 3 m a r[c p 14] / 46, X Y[11]\end{array}$ & Induction \\
\hline 11 & $\begin{array}{l}\text { Loss of } 1 p \text { and } \\
\text { gain of } 1 q\end{array}$ & $\begin{array}{l}\text { 1p13-p21 } \\
\text { 1q12 }\end{array}$ & $\begin{array}{l}43,-X, \operatorname{der}(\mathrm{X}) \mathrm{t}(\mathrm{X} ; 5)(\mathrm{q} 22 ; \mathrm{p} 13), \operatorname{del}(1) \\
(\mathrm{p} ? 21 \mathrm{p} ? 13), \\
\operatorname{add}(4)(\mathrm{p} 13), \operatorname{der}(5) \mathrm{t}(\mathrm{X} ; 5)(\mathrm{q} 22 ; \mathrm{p} 13) \\
\operatorname{add}(5)(\mathrm{q} 31), \operatorname{del}(6)(\mathrm{q} ? 21 \mathrm{q} 2 ? 5), \operatorname{der}(7) \\
\mathrm{t}(1 ; 7)(\mathrm{q} 12 ; \mathrm{p} 15) \\
\mathrm{t}(7 ; 21)(\mathrm{q} 11.2 ; \mathrm{q} 22), \operatorname{add}(9)(\mathrm{q} 34),- \\
10, \operatorname{del}(13)(\mathrm{q} 12 \mathrm{q} 14),-14, \operatorname{add}(14) \\
(\mathrm{q} ? 24), \operatorname{del}(18)(\mathrm{q} 21),-20, \operatorname{der}(21) \mathrm{t}(7 ; 21) \\
(\mathrm{q} 11.2 ; \mathrm{q} 22),+\operatorname{mar}[6] / \\
86, \text { idem } \times 2[2] / 46, X X[22]\end{array}$ & Induction \\
\hline
\end{tabular}


Citation: Scott EC, Chen Y, Chen Al, Smith SD, Barkay I, et al. (2015) Chromosome 1 Abnormalities Predict Shortened Progression Free and Overall Survival in Patients with High Risk Multiple Myeloma Undergoing Autologous Hematopoietic Cell Transplantation, a Retrospective Analysis. J Blood Lymph 5: 131. doi:10.4172/2165-7831.1000131

Page 4 of 7

\begin{tabular}{|c|c|c|c|c|}
\hline 12 & Loss of $1 p$ & $\begin{array}{l}1 \mathrm{p} 13 \\
1 \mathrm{p} 13\end{array}$ & $\begin{array}{l}56, X X,+\operatorname{add}(1)(p 13),+\operatorname{add}(1) \\
(p 13),+3,+5,+5,+6,+7,- \\
8,+9,+9,+\operatorname{add}(11)(\mathrm{p} 11.2),-13,- \\
14,+15,-16,+19,-22,+ \text { mar1, +mar2,+m } \\
\text { ar3[2]/46,XX[23] }\end{array}$ & Induction \\
\hline 13 & Gain of $1 q$ & $1 q 10$ & $\begin{array}{l}\text { 41,X,-Y,i(1)(q10), der(2)t(2;7) } \\
\text { (p?23;p?15), } \\
\text { add(4)(q25),add(4)(p?12), add(5) } \\
\text { (q 31),--7,-8,add(12)(q15),+add(12) } \\
\text { (q15),-13,-14,add(15)(q26), add(15) } \\
\text { (p11),-16,del(16)(p13),-20,+mar[1 } \\
\text { j/41,X,-Y, i(1)(q10), der(2)t(2;7) } \\
\text { (p?23;p?15),add(4)(q25), } \\
\text { add(4)(p?12),add(5)(q31),- } \\
\text { 7,-8,del(11)(q21), add(11) } \\
\text { (q?23),add(12)(q?15),add(15) } \\
\text { (q26),add(15)(p11),del(16)(p13),- } \\
\text { 16,del(19)(p11),del(19)(q13), add(20) } \\
\text { (p11)+mar[cp2]/46,XY[19] }\end{array}$ & Salvage \\
\hline 14 & $\begin{array}{l}\text { Gain of } 1 q \\
\text { and balanced } \\
\text { translocation }\end{array}$ & $\begin{array}{l}1 q 24 \\
t(1 ; 8)\end{array}$ & $\begin{array}{l}52, X, \operatorname{add}(X)(q 2 ? 6), t(1 ; 8) \\
(p ? 21 ; q 24),+5,+6, \\
\text { del(6)(q13q23) } \\
\text { x2,+7,+9,+15,+19[cp6]/60,XX,t }(1 ; 8) \\
(p ? 21 ; q 24),+\operatorname{der}(1) t(1 ; 8)(p ? 21 ; q 24),+ \\
2,+3,+5,+6,+7,+\operatorname{add}(7)(p 1 ? 3),+9,+9,+ \\
15,+15,+18,+19,+20[3] / \\
46, X X[23]\end{array}$ & Salvage \\
\hline 15 & $\begin{array}{l}\text { Gain of } 1 q \text { and } \\
\text { Gain of } 1 p\end{array}$ & $\begin{array}{l}1 \mathrm{q} 10 \\
1 \mathrm{p} 10\end{array}$ & $\begin{array}{l}\text { 40 46,-X,add(X)(q25),+1, dic(1;4) } \\
\text { (p33;q31),ins(1;?)(q25;?), } \\
\text { del(6)(q21),del(8)(q?22), } \\
\text { del(11)(q13q23),add(11) } \\
\text { (q23),-13,-16,add(17)(q23),-18,- } \\
\text { 22,+1 5mar[cp11]/46,XX[15] }\end{array}$ & Induction \\
\hline
\end{tabular}

Table 2: Chromosome 1 abnormalities. $N=15$ (6 induction, 9 salvage). Gains or losses of long or short arms of chromosome 1 and balanced translocations.

\begin{tabular}{|c|c|c|}
\hline Sample size & 169 & Salvage \\
\hline $\begin{array}{c}\text { Median overall } \\
\text { survival }\end{array}$ & $\begin{array}{c}57.0 \text { months (95\% Cl: } 44.3, \\
70.3)\end{array}$ & 30.0 months $(95 \% \mathrm{Cl}: 21.4, \ldots)^{*}$ \\
\hline Median PFS & $\begin{array}{c}27.3 \text { months (95\% Cl: } 20.1, \\
37.8)\end{array}$ & 18.1 months $(95 \% \mathrm{Cl}: 10.8,21.9)$ \\
\hline Relapse rate & $\begin{array}{c}52.48 \%(95 \% \mathrm{Cl}: 43.9,60.9 ; \\
\mathrm{n}=141)\end{array}$ & $59.6(95 \% \mathrm{Cl}: 45.1,73.0 ; \mathrm{n}=52)$ \\
\hline $\begin{array}{c}\text { Overall } \\
\text { response }\end{array}$ & $82.3 \%(95 \% \mathrm{Cl}: 75.6,87.7)$ & $77.8 \%(95 \% \mathrm{Cl}: 65.5,87.3)$ \\
\hline 2-year PFS & $65.1 \%(95 \% \mathrm{Cl}: 57.4,72.3)$ & $50.8 \%(95 \% \mathrm{Cl}: 37.9,63.6)$ \\
\hline 5-year PFS & $53.9 \%(95 \% \mathrm{Cl}: 46.0,61.5)$ & $46.0 \%(95 \% \mathrm{Cl}: 33.4,59.1)$ \\
\hline 2-year OS & $84.0 \%(95 \% \mathrm{Cl}: 77.6,89.2)$ & $74.6 \%(95 \% \mathrm{Cl}: 62.1,84.7)$ \\
\hline 5-year OS & $70.4 \%(95 \% \mathrm{Cl}: 62.9,77.2)$ & $66.7 \%(95 \% \mathrm{Cl}: 53.7,78.1)$ \\
\hline
\end{tabular}

Table 3: Response rates and survival outcomes in subjects with high risk features that received an autologous stem cell transplant divided by indication: induction and salvage. *Indicates that upper bound of the confidence interval is not computable.

Durie (SD)stage III (HR 2.50; $\mathrm{p}=0.0538$; 95\% CI: $0.985-6.349$ ) to be significant factors.

Similarly these factors were significantly associated with shortened OS214 chromosome 1 (HR 21.218; $\mathrm{p}=0.0002$; 95\% CI: 4.311-104.435) and S-D stage III (HR 4.212; $\mathrm{p}=0.0264 ; 95 \%$ CI:1.184- 14.983).

The final multivariable model for the induction cohort found that none of the potential risk factors independently or jointly predict shortened PFS or OS.

\section{Gene Variable}

As the ch 1 abnormality is significantly associated with both PFS and OS, we created a variable named 'gene variable' consisting of the following groups: 1$)$ no cytogenetic or FISH abnormality; 2$) \mathrm{t}(4 ; 14)$ OR del 17p OR cytogenetically detected del 13q OR OR $t(14 ; 16)$ OR $t(14 ; 20)$ a combination of these abnormalities; 3$)$ ch 1 abnormality alone OR with any other abnormality except del $13 \mathrm{q}$ and 4 ) the occurrence of both a ch1 and del 13q abnormality.

We compared the PFS and OS of these 4 groups. As there is no significant interaction present between gene variable and induction type, we report the aggregated association between the gene variable and survival outcome (PFS and OS).

Those with both a ch1 abnormality and 229 cytogenetically detected del 13q had the shortest median PFS and OS followed by those with a ch1 abnormality alone or with 230 another genetic abnormality, followed by group 2, followed by those without any cytogenetic or FISH abnormality.

The log rank test for PFS and OS across gene variable groups both had a $\mathrm{p}$ value of $<0.0001$ (Table 5 and Figures 1 and 2).

\section{Univariable and multivariable analyses of high risk factors associated with overall response rate and relapse rate}

The only covariate that independently predicts lower overall response was achieving less than a PR with 2 consecutive lines of therapy prior to transplant ( $\mathrm{p}=0.0013$, OR $=0.196,95 \%$ CI: 0.072 0.527 . Similarly, the only covariate that is independently significantly predicts higher relapse rate was achieving less than a PR with 2 consecutive lines of therapy prior to transplant $(\mathrm{p}=0.0391, \mathrm{OR}=5.031$, 95\% CI: 1.084-23.339. Lacking significant predictors, we did not final

\begin{tabular}{|c|c|c|c|c|}
\hline \multirow[b]{2}{*}{ High risk factor } & \multicolumn{2}{|r|}{ PFS } & \multicolumn{2}{|r|}{ os } \\
\hline & $\begin{array}{c}\mathrm{Pr}> \\
\text { ChiSq (p } \\
\text { value) }\end{array}$ & $\begin{array}{c}\text { Hazard Ratio (95\% } \\
\text { Hazard Ratio CI } \\
\text { limits) }\end{array}$ & $\begin{array}{l}\text { Pr > } \\
\text { ChiSq } \\
(p \\
\text { value })\end{array}$ & $\begin{array}{l}\text { Hazard Ratio } \\
\text { (95\% Hazard } \\
\text { Ratio Cl limits) }\end{array}$ \\
\hline $\begin{array}{c}\text { Chromosome } 1 \\
\text { abnormality }\end{array}$ & 0.0099 & $2.652(1.264-5.566)$ & 0.0002 & $\begin{array}{c}4.788(2.098- \\
10.928)\end{array}$ \\
\hline $\begin{array}{l}\text { Increased number } \\
\text { of prior therapies }\end{array}$ & 0.1852 & $1.081(0.963-1.214$ & 0.0539 & $\begin{array}{c}1.135(0.998- \\
1.292)\end{array}$ \\
\hline $\begin{array}{c}\leq \text { PR with } 2 \\
\text { consecutive lines } \\
\text { of therapy }\end{array}$ & 0.0172 & $2.142(1.145-4.007)$ & 0.0416 & $\begin{array}{l}2.148(1.030- \\
4.481)\end{array}$ \\
\hline $\begin{array}{c}\text { Multiple extra } \\
\text { medullary } \\
\text { plasmacytomas }\end{array}$ & 0.6193 & $1.232(0.540-2.810)$ & 0.2503 & $\begin{array}{c}1.814(0.657- \\
5.011)\end{array}$ \\
\hline ISS Stage & 0.7221 & & 0.47 & \\
\hline $\begin{array}{l}\text { ISS stage } 2 \text { vs. } \\
\text { stage } 1\end{array}$ & 0.6933 & $0.903(0.543-1.502)$ & 0.2408 & $\begin{array}{c}0.674(0.349- \\
1.303\end{array}$ \\
\hline $\begin{array}{l}\text { ISS stage } 3 \text { vs. } \\
\text { stage } 1\end{array}$ & 0.4201 & $0.768(0.405-1.458)$ & 0.421 & $\begin{array}{c}0.729(0.338- \\
1.573)\end{array}$ \\
\hline $\begin{array}{l}\text { Salmon - Durie } \\
\text { Stage }\end{array}$ & 0.5806 & & 0.996 & \\
\hline $\begin{array}{c}\text { Salmon - Durie } \\
\text { stage } 2 \text { vs. stage } 1\end{array}$ & 0.3001 & $2.885(0.389-21.399)$ & 0.9859 & * \\
\hline $\begin{array}{c}\text { Salmon - Durie } \\
\text { stage } 3 \text { vs. stage } 1\end{array}$ & 0.3243 & $2.706(0.374-19.584)$ & 0.9859 & * \\
\hline Del $13 q$ cyto & 0.0608 & $1.756(0.975-3.164)$ & 0.216 & $\begin{array}{c}1.605(0.759- \\
3.396)\end{array}$ \\
\hline $\mathrm{t}(4 ; 14)$ & 0.9549 & $0.978(0.454-2.105)$ & 0.8969 & $\begin{array}{l}0.926(0.289- \\
2.965)\end{array}$ \\
\hline$t(14 ; 16)$ & 0.9824 & * & 0.989 & * \\
\hline del $17 p$ & 0.2344 & $1.741(0.698-4.342)$ & 0.0727 & $\begin{array}{c}2.607(0.916- \\
7.420)\end{array}$ \\
\hline $\begin{array}{l}\text { Plasmablastic } \\
\text { morphology }\end{array}$ & 0.2824 & $0.339(0.047-2.434)$ & 0.9826 & * \\
\hline
\end{tabular}

Table 4: Univariable analysis of high risk factors associated with shorter PFS and OS in subjects that received an auto-HCT for MM. *not computable due to high standard error. 
Citation: Scott EC, Chen Y, Chen AI, Smith SD, Barkay I, et al. (2015) Chromosome 1 Abnormalities Predict Shortened Progression Free and Overall Survival in Patients with High Risk Multiple Myeloma Undergoing Autologous Hematopoietic Cell Transplantation, a Retrospective Analysis. J Blood Lymph 5: 131. doi:10.4172/2165-7831.1000131

Page 5 of 7

\begin{tabular}{|c|c|c|c|}
\hline Group & Gene variable & Median PFS in months (95\% Cl) & Median OS in months (95\% Cl) \\
\hline 1 & No FISH or cytogenetic abnormality $(n=154)$ & $24.44(20.14,37.88)$ \\
\hline 2 & Del13q;t(4;14); $t(14 ; 16) ; t(14 ; 20)$ or del 17p $(n=38)$ & $26.58(18.10,45.47)$ \\
\hline 3 & Chromosome 1 alone or with any other genetic abnormality EXCEPT del13q $(n=8)$ & $16.79(6.60)$ & * \\
\hline 4 & Chromosome 1 and del13q by cytogenetics $(n=7)$ & $4.76(0.98,7.06)$ & $35.22(16.30,35.22)$ \\
\hline
\end{tabular}

Table 5: PFS and OS in months for each gene variable group. *The upper bound of $95 \% \mathrm{Cl}$ is not computable. " Median OS has not yet been reached.

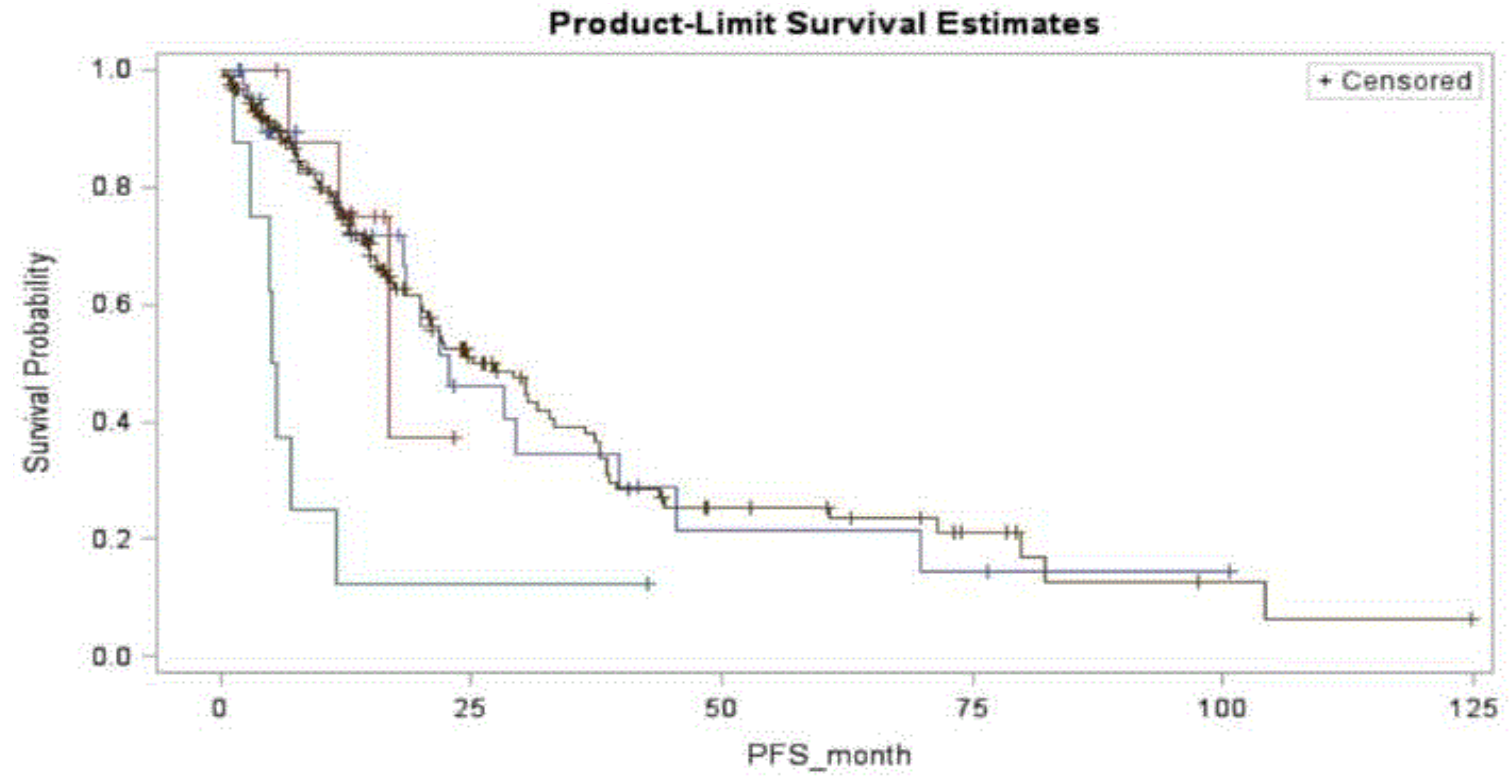

Figure 1: Progression free survival (PFS) comparison between the following groups: 1 ) no cytogenetic or FISH abnormality (brown); 2$) t(4 ; 14), t(14 ; 16), t(14 ; 20)$; del $17 \mathrm{p}$ or del $13 \mathrm{q}$ or a combination of these abnormalities (blue); 3) ch1 abnormality alone or with any other abnormality except del 13q (red) and 4 ) both a ch1 and del 13q abnormality (green).

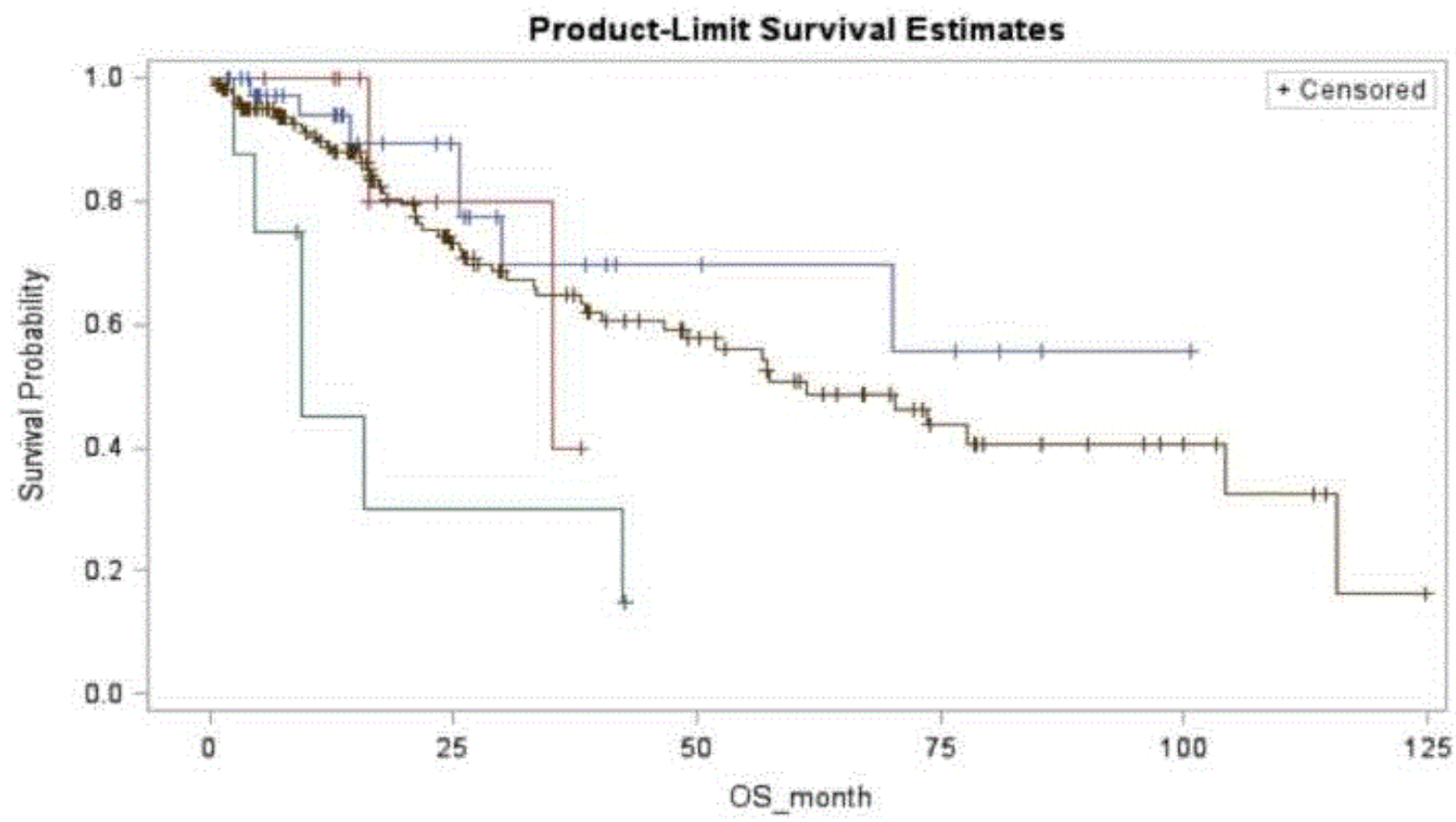

Figure 2: Overall survival (OS) comparison between the following groups: 1) no cytogenetic or FISH abnormality (brown); 2 ) $t$ (4;14), del 17 $p$ or del 13 q or a combination of these abnormalities (blue); 3) ch1 abnormality alone or with any other abnormality except del 13q (red) and 4 ) both a ch1 and del $13 q$ abnormality (green). 
Citation: Scott EC, Chen Y, Chen AI, Smith SD, Barkay I, et al. (2015) Chromosome 1 Abnormalities Predict Shortened Progression Free and Overall Survival in Patients with High Risk Multiple Myeloma Undergoing Autologous Hematopoietic Cell Transplantation, a Retrospective Analysis. J Blood Lymph 5: 131. doi:10.4172/2165-7831.1000131

Page 6 of 7

any multivariable models worth presenting for both overall response and relapse rate.

\section{Discussion}

In this study, we identified that abnormalities of chromosome 1 are significant risk factors associated with the shortest PFS and OS when compared to all other high risk MM groups, including those with other known high risk cytogenetic factors: deletion $17 \mathrm{p}, \mathrm{t}(4 ; 14), \mathrm{t}(14 ; 16)$, $\mathrm{t}(14 ; 20)$, deletion $13 \mathrm{q}$ (cytogenetic), elevated biomarkers (ISS), high risk clinical extra medullary disease and S-D staging) and morphologic (plasmablastic) features. Our gene risk model demonstrated that subjects with 253 the combination of a chromosome 1 abnormality and cytogenetically detected del $13 \mathrm{q}$ suffered from dismal outcomes (median PFS 4.76 and OS of 9.43 months) post auto-HCT, closely followed by those with chromosome 1 abnormalities alone or in combination with deletion 17p or $\mathrm{t}(4 ; 14)$ (median PFS 16.79; OS 35.22 months).

Those with any combination of other well-known high risk FISH or cytogenetic factors: del 13q, del $17 \mathrm{p}, \mathrm{t}(4 ; 14), \mathrm{t}(14 ; 16)$ and $\mathrm{t}(14 ; 20)$ had a median PFS of 26.58 months and OS of 35.22 months and those with no abnormalities had median PFS of 24.44 and OS of 57.04 months, both far superior than outcomes for patients with chromosome 1 abnormalities. Finally, despite the heterogeneity and the small number of patients with a chromosome labnormality in this cohort $(n=15)$ of high risk patients, we have shown using a multivariate model statistical significance of the association of chromosome 1 abnormalities with shortened PFS (HR of 22.93) and OS (HR of 21.218) particularly in those undergoing salvage auto-HCT. We acknowledge that the confidence intervals are very wide given the small number of patients.

The co-existence of gain in $1 \mathrm{q}$ with deletion $17 \mathrm{p}$ and $\mathrm{t}(4 ; 14)$, $\mathrm{t}(14 ; 16)$ and $\mathrm{t}(14 ; 20)$ and the accumulation of genomic abnormalities over time is recognized as very high risk disease with progressive impact on survival in a recent analysis of 1069 subjects in the MRC IX trial [19]. Interestingly, our data found that del 13q coexisted more commonly with chromosome 1 abnormalities and was significantly more impactful than when combined with other high risk genomic abnormalities. Our data also highlights the complexity of cytogenetic abnormalities in patients with multiple myeloma, and that abnormalities of chromosome 1 are often accompanied by a myriad of other chromosomal alterations that may evolve over time.

The predominance of genes located on chromosome 1 in the highrisk gene expression profile score and the observation that an increase in copy number of 1q21 are associated with relapsed disease 7 raises the question whether aberrations of chromosome 1 are a cause or a consequence of disease progression. Recent evidence shows that the jumping translocation 1q12 is a mechanism for the simultaneous gain of 1q21 and deletion of $17 \mathrm{p}$ in cytogenetically defined high-risk disease [20]. Possible biological reasons explaining this observation based upon translational studies are lacking. The cellular and molecular mechanisms of chromosome 1 abnormalities are not well understood but have been proposed to involve decondensation of pericentromeric heterochromatin.1 Chromosomal instability leading to tumor progression through silencing tumor suppressor genes has also been proposed as a mechanism [21].

The only other factor that was independently significantly associated with shortened PFS and OS in the univariable analysis of the entire cohort was achieving less than a PR with 2 consecutive lines of therapy prior to transplant. No particular high risk factor was identified to be independently or jointly associated with PFS or OS for induction patients. However, for patients receiving salvage HCT, having a chromosome 1 abnormality and a higher Salmon Durie stage ( 3 vs. 2 ) are predictive of a shorter PFS and OS. It should be noted that we included MM subjects with any high risk feature that received an auto-HCT as part of their induction and salvage treatment, and thus the outcome measures for the entire cohort are expectedly low due to the exclusion of standard or good risk subjects. In the induction cohort the median PFS and OS are 27.3 and 57.0 months respectively, with 2 and 5 year PFS rates of $65.1 \%$ and $53.9 \%$ and OS rates of $84.0 \%$ and $70.4 \%$ respectively, which are somewhat lower to recently published large studies of auto-HCT as consolidation after primary therapy which demonstrate median time to progression of 21-46 months after single autologous transplant, depending on the addition of lenalidomide maintenance [22-24].

These studies (in which patients in the current cohort have participated) have established expected PFS and OS benchmarks for initial auto-HCT that should identify higher risk subsets in the modern era. The median PFS and OS of 18.1 and 30 months in our salvage auto-HCT are comparable to other studies that included all risk groups $[25,26]$. The past two decades have produced major advances in the treatment of MM, which have led to improvements in response rates and survival for patients with this incurable malignancy. Despite these advances in novel therapies and refinement of stem cell transplant procedures; certain patients still suffer from aggressive disease and succumb to poor outcomes. Efforts to identify particular sub-groups of patients, based on prognostic clinical, biologic and cytogenetic risk factors, which may benefit from particular treatment approaches, are needed. While proteasome inhibitors and immunomodulatory agents have been shown to overcome the poor prognostic impact of certain higher risk genomic abnormalities these therapies have not yet been shown to overcome the poor prognosis of chromosome 1 abnormalities [27]. Given the small number of patients with chromosome 1 abnormalities and the heterogeneity of pre-treatment and maintenance approaches in this cohort, it was not possible to evaluate the impact of specific abnormalities of chromosome 1 or of particular treatments on auto-HCT outcomes.

In summary, we suggest that the presence of a chromosome 1 abnormality, in particular in combination with deletion $13 \mathrm{q}$ is a very significant risk factor for short remission duration and overall survival after salvage auto-HCT. Novel induction, transplant, and maintenance approaches need to be considered for this group. Future investigations should be aimed at discovering the true incidence of these abnormalities in large prospective cohorts, the significance of each individual chromosome 1 abnormality (losses, gains, and translocations with various partners) and the impact of particular treatment strategies on overcoming these poor prognostic factors.

\section{Clinical practice points}

Controversy exists regarding the impact of various abnormalities of chromosome 1 in multiple myeloma

In this real world cohort of heterogeneously treated patients, chromosome 1 abnormalities significantly impacted PFS and OS in patients undergoing salvage auto-HCT

Future studies are needed to further characterize different chromosome 1 abnormalities and their impact on treatment outcomes.

\section{Acknowledgement}

Research support was awarded to Emma C. Scott from Millenium Takeda to finance biostatistics work. 
Citation: Scott EC, Chen Y, Chen Al, Smith SD, Barkay I, et al. (2015) Chromosome 1 Abnormalities Predict Shortened Progression Free and Overall Survival in Patients with High Risk Multiple Myeloma Undergoing Autologous Hematopoietic Cell Transplantation, a Retrospective Analysis. J Blood Lymph 5: 131. doi:10.4172/2165-7831.1000131

Emma C. Scott designed the research study, Emma C. Scott, Andy I. Chen, Stephen D. Smith, Ido Barkay, Will Dibb, James Dibb, Rachel Frires, Matthew Siegel, Eva Medvedova, Phoebe Trubowitz, and Richard T. Maziarz performed the research study, Emma C. Scott, Alex Stentz, Yiyi Chen analyzed the data, Emma C. Scott, Yiyi Chen and Richard T. Maziarz wrote the paper.

\section{References}

1. Sawyer JR, Swanson CM, Koller MA, North PE, Ross SW (1995) Centromeric instability of chromosome 1 resulting in multibranched chromosomes, telomeric fusions, and "jumping translocations" of $1 \mathrm{q}$ in a human immunodeficiency virusrelated non-Hodgkin's lymphoma. Cancer 76: 1238-1244.

2. Sawyer JR, Tricot G, Mattox S, Jagannath S, Barlogie B (1998) Jumping translocations of chromosome $1 \mathrm{q}$ in multiple myeloma: evidence for a mechanism involving decondensation of pericentromeric heterochromatin. Blood 91: 1732-1741.

3. Shaughnessy J (2005) Amplification and overexpression of CKS1B at chromosome band 1q21 is associated with reduced levels of p27Kip1 and an aggressive clinical course in multiple myeloma. Hematology 10 Suppl 1: 117126.

4. Hanamura I, Stewart JP, Huang Y, Zhan F, Santra M, et al. (2006) Frequent gain of chromosome band 1q21 in plasma-cell dyscrasias detected by fluorescence in situ hybridization: incidence increases from MGUS to relapsed myeloma and is related to prognosis and disease progression following tandem stem-cell transplantation. Blood 108: 1724-1732.

5. Chang $\mathrm{H}, \mathrm{Qi} X$, Trieu $\mathrm{Y}, \mathrm{Xu}$ W, Reader JC, et al. (2006) Multiple myeloma patients with CKS1B gene amplification have a shorter progression-free survival post-autologous stem cell transplantation. $\mathrm{Br} \mathrm{J}$ Haematol 135: 486491.

6. Fonseca R, Van Wier SA, Chng WJ, Ketterling R, Lacy MQ, et al. (2006) Prognostic value of chromosome 1q21 gain by fluorescent in situ hybridization and increase CKS1B expression in myeloma. Leukemia 20: 2034-2040.

7. Shaughnessy JD Jr, Zhan F, Burington BE, Huang Y, Colla S, et al. (2007) A validated gene expression model of high-risk multiple myeloma is defined by deregulated expression of genes mapping to chromosome 1. Blood 109: 2276-2284.

8. Chang H, Trieu Y, Qi X, Jiang NN, Xu W, et al. (2011) Impact of cytogenetics in patients with relapsed or refractory multiple myeloma treated with bortezomib: Adverse effect of 1q21 gains. Leuk Res 35: 95-98.

9. Fonseca R, Bergsagel PL, 388 Drach J, Shaughnessy J, Gutierrez N, et al. (2009) International Myeloma Working Group molecular classification of multiple myeloma: spotlight review. Leukemia 23: 2210-2221.

10. Kumar SK, Mikhael JR, Buadi FK, Dingli D, Dispenzieri A, et al. (2009) Management of newly diagnosed symptomatic multiple myeloma: updated Mayo Stratification of Myeloma and Risk-Adapted Therapy (mSMART) consensus guidelines. Mayo Clinic proceedings. Mayo Clinic 84: 1095-1110.

11. http://www.nccn.org/professionals/physician_gls/pdf/myeloma.pdf

12. Avet-Loiseau H, Attal M, Moreau P, Charbonnel C, Garban F, et al. (2007) Genetic abnormalities and survival in multiple myeloma: the experience of the Intergroupe Francophone du Myélome. Blood 109: 3489-3495.

13. Boyd KD, Ross FM, Walker BA, Wardell CP, Tapper WJ, et al. (2011) Mapping of chromosome $1 \mathrm{p}$ deletions in myeloma identifies FAM46C at $1 \mathrm{p} 12$ and CDKN2C at $1 \mathrm{p} 32.3$ as being genes in regions associated with adverse survival. Clin Cancer Res 17: 7776-7784.

14. Qazilbash MH, Saliba RM, Ahmed B, Parikh G, Mendoza F, et al. (2007) Deletion of the short arm of chromosome 1 (del 1p) is a strong predictor of poor outcome in myeloma patients undergoing an autotransplant. Biol Blood Marrow Transplant 13: 1066-1072.

15. Hadwiger JA, Wittenberg $C$, Richardson HE, de Barros Lopes M, Reed S (1989) A family of cyclin homologs that control the G1 phase in yeast. Proc Natl Acad Sci U S A 86: 6255-6259.

16. Ganoth D, Bornstein G, Ko TK, Larsen B, Tyers M, et al. (2001) The cell-cycle regulatory protein $\mathrm{Cks} 1$ is required for $\mathrm{SCF}(\mathrm{Skp2})$-mediated ubiquitinylation of p27. Nat Cell Biol 3: 321-324.

17. Rosiñol L, Carrió A, Bladé J, Queralt R, Aymerich M, et al. (2005) Comparative genomic hybridisation identifies two variants of smoldering multiple myeloma. Br J Haematol 130: 729-732.

18. Kaplan EaM P (1958) Nonparametric estimation from incomplete observations. Journal of the American Statistical Association 53: 457-481.

19. Boyd KD, Ross FM, Chiecchio L, Dagrada GP, Konn ZJ, et al. (2012) A nove prognostic model in myeloma based on co-segregating adverse FISH lesions and the ISS: analysis of patients treated in the MRC Myeloma IX trial. Leukemia 26: 349-355.

20. Sawyer JR, Tian E, Heuck CJ, Epstein J, Johann DJ, et al. (2014) Jumping translocations of $1 q 12$ in multiple myeloma: a novel mechanism for deletion of $17 p$ in cytogenetically defined high-risk disease. Blood 123: 2504-2512.

21. Lengauer C, Kinzler KW, Vogelstein B (1998) Genetic instabilities in human cancers. Nature 396: 643-649.

22. Krishnan A, Pasquini MC, Logan B, Stadtmauer EA, Vesole DH, et al. (2011) Autologous haemopoietic stem-cell transplantation followed by allogeneic or autologous haemopoietic stem-cell transplantation in patients with multiple myeloma (BMT CTN 0102): a phase 3 biological assignment trial. Lancet Oncol 12: $1195-1203$.

23. McCarthy PL, Owzar K, Hofmeister CC, Hurd DD, Hassoun H, et al. (2012) Lenalidomide after stem-cell transplantation for multiple myeloma. $\mathrm{N}$ Engl $\mathrm{J}$ Med 366: 1770-1781.

24. Attal M, Harousseau JL (2007) Role of autologous stem-cell transplantation in multiple myeloma. Best Pract Res Clin Haematol 20: 747-759.

25. Auner HW, Szydlo R, Rone A, Chaidos A, Giles C, et al. (2013) Salvage autologous stem cell transplantation for multiple myeloma relapsing or progressing after up-front autologous transplantation. Leuk Lymphoma 54 2200-2204.

26. Gonsalves WI, Gertz MA, Lacy MQ, Dispenzieri A, Hayman SR, et al. (2013) Second auto-SCT for treatment of relapsed multiple myeloma. Bone Marrow Transplant 48: 568-573.

27. Biran N, Malhotra J, Bagiella E, Cho HJ, Jagannath S, et al. (2014) Patients with newly diagnosed multiple myeloma and chromosome 1 amplification have poor outcomes despite the use of novel triplet regimens. Am J Hematol 89: 616-620. 